Série dos Seminários de Acompanhamento à Pesquisa

\title{
Prediction of Length of Stay in Intensive Care Units
}

\author{
Autor(es): \\ Igor Tona Peres
}




\title{
Série dos Seminários de Acompanhamento à Pesquisa
}

\section{Prediction of Length of Stay in Intensive Care Units}

\author{
Autor(es): \\ Igor Tona Peres
}

CRÉDITOS:

SISTEMA MAXWELL / LAMBDA

https://www.maxwell.vrac.puc-rio.br/

Organizadores: Fernanda Baião / Soraida Aguilar 


\section{Igor Tona Peres}

PhD Student

Advisor: Fernando Luiz Cyrino Oliveira

Co-advisor: Silvio Hamacher

External advisor: Fernando Bozza

External advisor: Jorge Salluh 


\section{Introduction}

- Studies have shown that a small percentage of ICU patients presented a prolonged LoS.

- However, those few patients account for a large proportion of ICU days.

- Therefore, the early identification of prolonged stay patients can assist in improving unit efficiency.

- The main reasons for hospital administrators to predict ICU LOS are threefold:

- (i) planning the number of ICU resources required;

- (ii) identifying patients with greater risk of prolonged stay aiming to drive quality improvement actions;

- (iii) enabling case-mix adjustments for benchmarking analysis. 


\section{Objectives}

- To present a methodology that makes possible to predict the patient's length of stay in the Intensive Care Unit.

- Specific goals:

- To propose and apply a data-driven methodology to predict the ICU LoS at day one

- To dynamic adjust the prediction for the next seven days 


\section{Methodology}

- Figure 1 presents the framework of the proposed methodology, which was adapted from Dantas et al. (2020):

\begin{tabular}{|c|c|c|c|}
\hline Data Preparation & Visualization and Data & Data Splitting & Data Preprocessing \\
\hline $\begin{array}{ll}\text { - Import database } \\
\text { - } & \text { Feature engineering } \\
\end{array}$ & $\begin{array}{ll}\text { - } & \text { Missing values } \\
\text { - } & \text { Descriptive } \\
\text { statistical analysis } \\
\text { - Outliers detection } \\
\text { and treatment }\end{array}$ & & $\begin{array}{ll}\text { - } & \text { Dimension Reduction } \\
\text { - } & \text { Imputation } \\
\text { - } & \text { Feature Selection } \\
& \text { Resolve Skewnestons }\end{array}$ \\
\hline
\end{tabular}

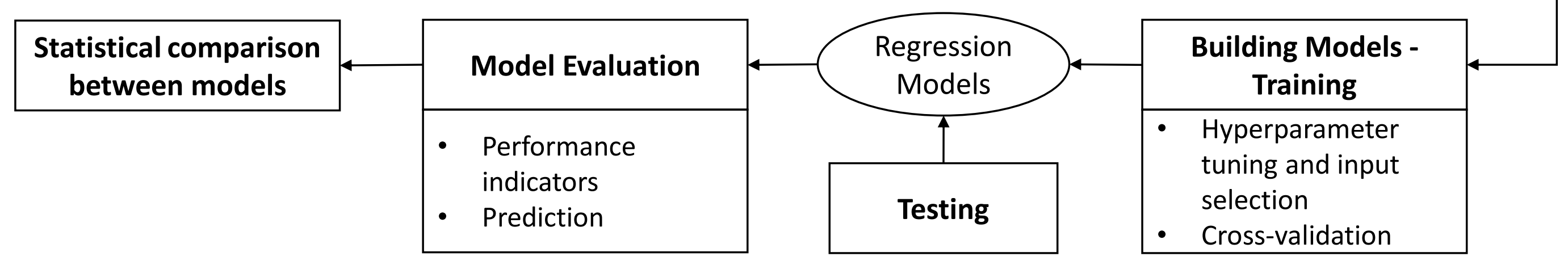




\section{Dataset description}

- The data represents a set of 113 mixed-type ICUs from 40 different Brazilian hospitals.

- Total of 100,245 independent admissions from January 01 to December 31, 2019.

- The complete dataset is a join of six tables:

- Sheet 2: Demographic data

- Sheet 3: Comorbidities

- Sheet 5: ICU complications ("first 24h")

- Sheet 6: Physiological and laboratory data ("first $1 h$ ")

- Sheet 8: Secondary diagnosis 


\section{Dataset description}

- Initital dataset =103,195 independent admissions

- Final dataset $=100,340$ independent admissions

- Filters:

- Patients $>=16$ and $<=115$ years old (518 removed)

- ICU LOS $>=0$ Oh (108 removed)

- ICU LoS >= 6 h ( 858 removed)

- Previous hospital LoS lower than 60 days (876 removed)

- Unit admission date $>=$ Hospital admission date (7 removed)

- Presenting the admission main diagnosis code (1336 removed) 


\section{Data Preparation}

- Feature engineering

Adjusting existing features:

- Adjust the hospital length of stay prior to unit admission (using the dates)

- When ChronicHealthStatusName has value, all not informed comorbidities can be replaced by "No"

- Laboratorial and physiological features were changed

- Gender "undefined" was replaced by "Not informed" and then will be imputed.

- Admission source was reclassified

- Surgical center; Ward/Room/Semi-intensive Unit; Home-care/Transfers/Others 


\section{Data Preparation}

- Feature engineering

Excluding organizational features

- ICU Type

- UnitCode

- HospitalCode

- Beds

- HealthInsuranceName 


\section{Data Preparation}

- Feature engineering

Propose new features from existing ones:

- Number of first day complications ("n_complication")

- Presence of any first day complication ("has_complication")

Treatment for "Secondary Diagnosis"

- Only diagnosis with more than 400 registries 


\section{Visualization and Data Cleaning}

- Missing values

- Analyze the behavior of the missing data.

- Features with more than 30\% of missing will be excluded from the analysis (White et al., 2011).

- Data with less than $30 \%$ of missing will be imputed. 


\section{Results - Missing Data}

- Features with more than $30 \%$ of missing:

- Demographic data

- ICDCode (100\%);

- Physiological and laboratory data ("first 24h")

- LowestPaCO21h (94\%); LowestPaO21h (94\%); LowestFiO21h (92\%); HighestPaO21h (75\%); HighestPaCO21h (75\%); HighestFiO21h (61\%);

- Some relevant features will not be removed now:

- $\mathrm{PaO} 2 \mathrm{FiO} 2$ (83.4\%); PH (74.5\%); Lactate (65\%); Bilirubin (57.5\%); BMI (41\%) 


\section{Visualization and Data Cleaning}

- Descriptive statistical analysis

- We will apply a univariate analysis between the explicative variables and the ICU LoS.

- Pearson Correlation for numerical variables

- Cramer's V for categorical ones 


\section{Results - Descriptive analysis}

- Correlation with LoS for numeric variables

\begin{tabular}{lr} 
Feature & Correlation \\
\hline Saps3DeathProbabilityStandardEquation & 0.14 \\
Saps3Points & 0.14 \\
LowestGlasgowComaScale1h & -0.13 \\
SofaScore & 0.12 \\
LengthHospitalStayPriorUnitAdmission & 0.09 \\
n_complication & 0.09 \\
MFIpoints & 0.09 \\
MFIScore & 0.09 \\
Age & 0.07 \\
HighestRespiratoryRate1h & 0.07 \\
CharlsonComorbiditylndex & 0.06 \\
HighestHeartRate1h & 0.05 \\
BUN & 0.04 \\
Urea & 0.04 \\
PaO2FiO2 & 0.02 \\
HighestTemperature1h & 0.02 \\
HighestCreatinine1h & 0.02 \\
LowestDiastolicBloodPressure1h & -0.02 \\
HighestLeukocyteCount1h & 0.01 \\
PH & 0.01 \\
LowestPlateletsCount1h & 0.01 \\
BMl & -0.01 \\
Bilirubin & 0.01 \\
LowestMeanArterialPressure1h & -0.01 \\
Lactate & 0.00 \\
LowestSystolicBloodPressure1h & 0.00
\end{tabular}




\section{Results - Descriptive analysis}

- Correlation with LoS for categorical variables

\begin{tabular}{lr} 
Feature & Correlation \\
\hline AdmissionMainDiagnosisName & 0.29 \\
IsMechanicalVentilation & 0.26 \\
IsVasopressors & 0.22 \\
has_complication & 0.22 \\
\hline AdmissionReasonName & 0.20 \\
\hline AdmissionSourceName & 0.17 \\
\hline IsRespiratoryFailure & 0.17 \\
IsDementia & 0.16 \\
FrailPatientMFI & 0.15 \\
IsNonInvasiveVentilation & 0.13 \\
IsArterialHypertension & 0.13 \\
IsSevereCopd & 0.10 \\
IsCrf & 0.09 \\
IsStroke & 0.08 \\
IsReadmission & 0.08 \\
Sec_Sepseechoqueséptico & 0.08 \\
IsChronicAtrialFibrilation & 0.08 \\
IsRenalReplacementTherapy & 0.08 \\
IsAcuteKidneyInjury & 0.08
\end{tabular}




\section{Outlier detection and treatment}

- Dispersion/Boxplot analysis

- 376 values were considered outliers

- Outliers will be replaced by "NA" and then imputed

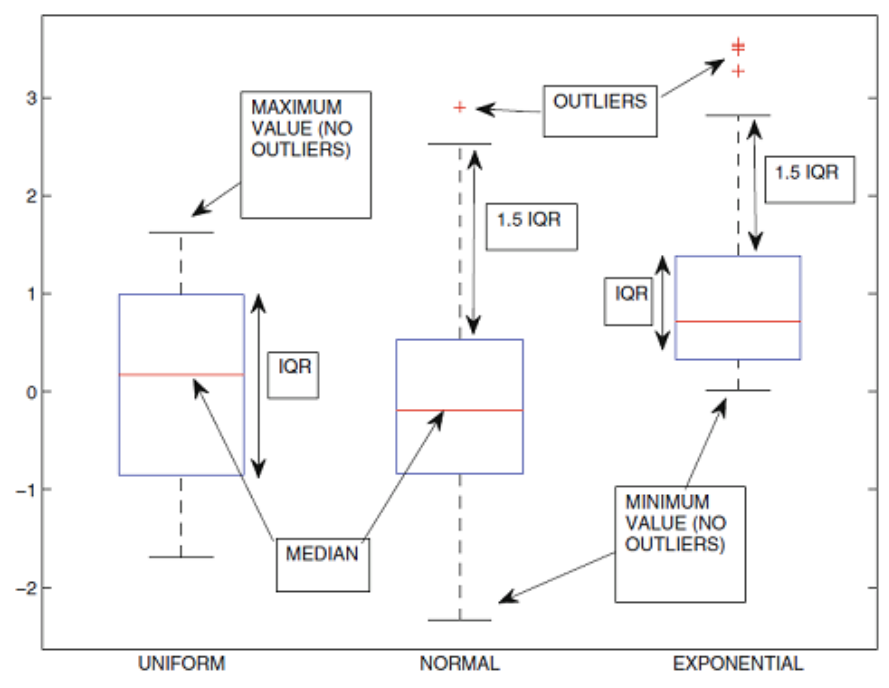




\section{Outlier detection and treatment}

- Histogram of ICU LoS

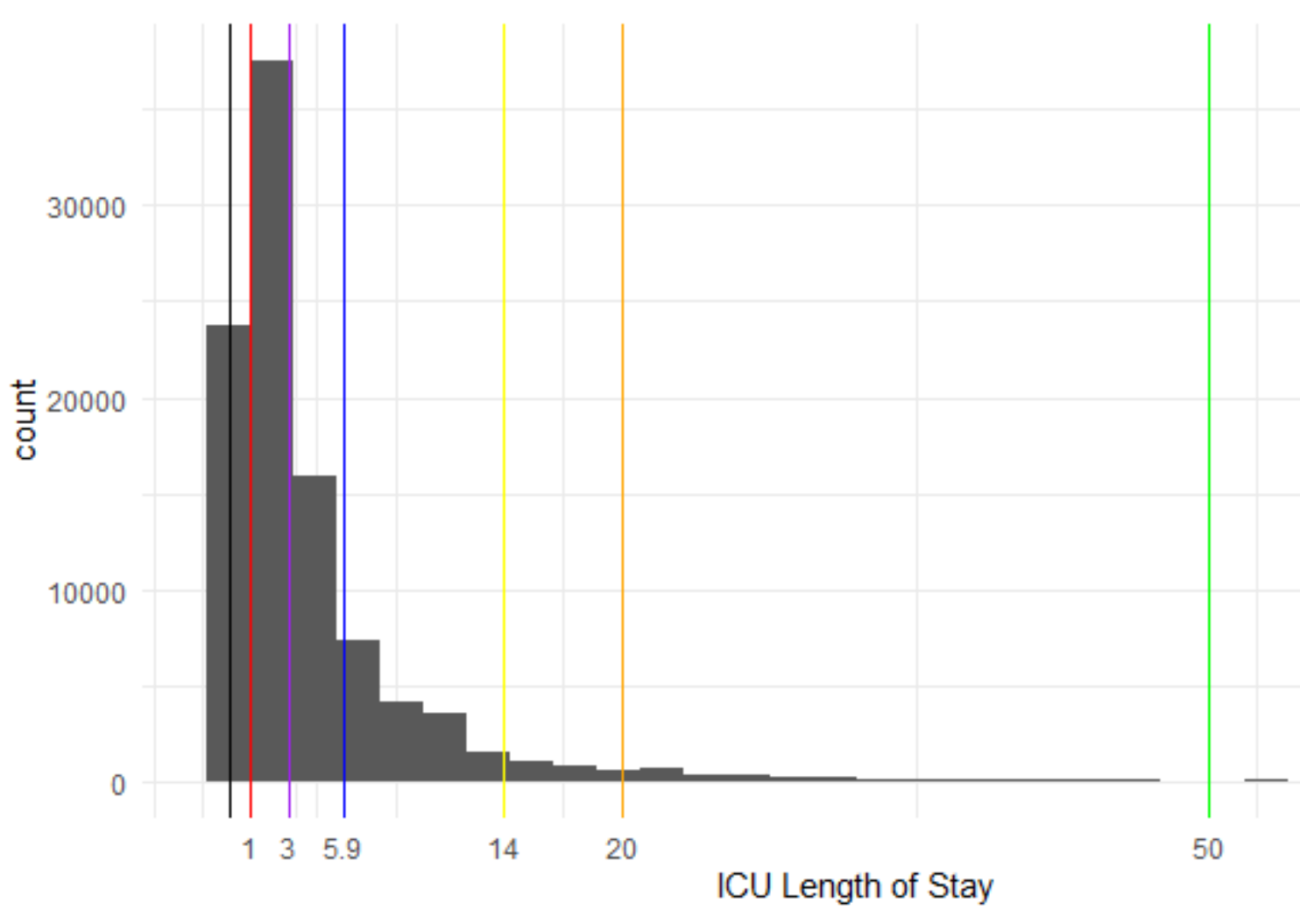




\section{Data Splitting}

- We will apply this sampling methodology, splitting $80 \%$ of the dataset for training and $20 \%$ for testing

- To provide an unbiased sense of model effectiveness, the predictive model should be evaluated on samples that were not used to build or tune the model.

- Training with 5-fold cross-validation 


\section{Data Preparation}

- Feature engineering

Grouping "Admission Main Diagnosis" (851 possible classes)

\begin{tabular}{|c|c|c|c|c|c|c|c|}
\hline Main Diagnosis Name & Total & $\%$ & $\begin{array}{c}\text { Cumulati } \\
\text { ve } \%\end{array}$ & $\begin{array}{l}\text { Mean } \\
\text { ICU LoS }\end{array}$ & $\begin{array}{l}\text { Median } \\
\text { ICU LoS }\end{array}$ & $\begin{array}{l}\text { Q90 ICU } \\
\text { LoS }\end{array}$ & $\begin{array}{c}\text { ICU } \\
\text { Mortality }\end{array}$ \\
\hline Pneumonia comunitária & 6941 & $8.7 \%$ & $8.7 \%$ & 8.4 & 4.0 & 18.0 & $10 \%$ \\
\hline Dor torácica & 4188 & $5.2 \%$ & $13.9 \%$ & 2.6 & 2.0 & 5.0 & $0 \%$ \\
\hline Infecção urinária sintomática, não especificada & 3387 & $4.2 \%$ & $18.1 \%$ & 6.7 & 4.0 & 13.0 & $6 \%$ \\
\hline Síncope & 2983 & $3.7 \%$ & $21.8 \%$ & 3.7 & 2.0 & 6.0 & $1 \%$ \\
\hline Angina instável & 2592 & $3.2 \%$ & $25.1 \%$ & 3.3 & 2.0 & 6.0 & $0 \%$ \\
\hline Insuficiência cardíaca aguda (descompensada) & 2112 & $2.6 \%$ & $27.7 \%$ & 7.6 & 5.0 & 15.9 & $7 \%$ \\
\hline AVC isquêmico & 1651 & $2.1 \%$ & $29.7 \%$ & 7.7 & 3.0 & 16.0 & $5 \%$ \\
\hline Epilepsia e transtornos convulsivos & 1599 & $2.0 \%$ & $31.7 \%$ & 5.2 & 3.0 & 10.0 & $3 \%$ \\
\hline Fibrilação atrial & 1351 & $1.7 \%$ & $33.4 \%$ & 4.0 & 2.0 & 8.0 & $3 \%$ \\
\hline Infarto miocárdico sem supra de ST & 1211 & $1.5 \%$ & $34.9 \%$ & 5.2 & 3.0 & 10.0 & $4 \%$ \\
\hline Tromboembolismo pulmonar & 1155 & $1.4 \%$ & $36.4 \%$ & 5.0 & 3.0 & 8.0 & $3 \%$ \\
\hline Intoxicações exógenas & 1007 & $1.3 \%$ & $37.6 \%$ & 2.5 & 2.0 & 5.0 & $0 \%$ \\
\hline Gastroplastias & 996 & $1.2 \%$ & $38.9 \%$ & 1.3 & 1.0 & 2.0 & $0 \%$ \\
\hline Acidente isquêmico transitório & 988 & $1.2 \%$ & $40.1 \%$ & 3.0 & 2.0 & 5.0 & $1 \%$ \\
\hline Gastroenterites / gastroenterocolites & 954 & $1.2 \%$ & $41.3 \%$ & 3.7 & 2.0 & 7.0 & $2 \%$ \\
\hline Outros diagnósticos, não classificados & 924 & $1.2 \%$ & $42.4 \%$ & 4.0 & 3.0 & 8.0 & $3 \%$ \\
\hline Hemorragia digestiva alta & 894 & $1.1 \%$ & $43.6 \%$ & 5.2 & 3.0 & 10.0 & $5 \%$ \\
\hline Outras complicações neurológicas & 854 & $1.1 \%$ & $44.6 \%$ & 4.6 & 2.0 & 8.0 & $3 \%$ \\
\hline DPOC descompensada & 853 & $1.1 \%$ & $45.7 \%$ & 7.8 & 5.0 & 16.0 & $8 \%$ \\
\hline
\end{tabular}




\section{Data Preparation}

- Feature engineering

Grouping "Admission - Main Diagnosis" (19 possible classes)

\begin{tabular}{lrrrrrrr}
\multicolumn{1}{c}{ Main Diagnosis Name } & Total & \multicolumn{1}{c}{$\begin{array}{c}\text { Cumulati } \\
\text { ve \% }\end{array}$} & \multicolumn{1}{c}{$\begin{array}{c}\text { Mean } \\
\text { ICU LoS }\end{array}$} & $\begin{array}{r}\text { Median } \\
\text { ICU LoS }\end{array}$ & $\begin{array}{c}\text { Q90 ICU } \\
\text { LoS }\end{array}$ & $\begin{array}{r}\text { ICU } \\
\text { Mortality }\end{array}$ \\
\hline Pneumonia comunitária & 6941 & $8.7 \%$ & $8.7 \%$ & 8.4 & 4.0 & 18.0 & $10 \%$ \\
\hline Dor torácica & 4188 & $5.2 \%$ & $13.9 \%$ & 2.6 & 2.0 & 5.0 & $0 \%$ \\
\hline Infecção urinária sintomática, não especificada & 3387 & $4.2 \%$ & $18.1 \%$ & 6.7 & 4.0 & 13.0 & $6 \%$ \\
\hline Síncope & 2983 & $3.7 \%$ & $21.8 \%$ & 3.7 & 2.0 & 6.0 & $1 \%$ \\
Angina instável & 2592 & $3.2 \%$ & $25.1 \%$ & 3.3 & 2.0 & 6.0 & $0 \%$ \\
\hline Insuficiência cardíaca aguda (descompensada) & 2112 & $2.6 \%$ & $27.7 \%$ & 7.6 & 5.0 & 15.9 & $7 \%$ \\
\hline AVC isquêmico & 1651 & $2.1 \%$ & $29.7 \%$ & 7.7 & 3.0 & 16.0 & $5 \%$ \\
\hline Epilepsia e transtornos convulsivos & 1599 & $2.0 \%$ & $31.7 \%$ & 5.2 & 3.0 & 10.0 & $3 \%$ \\
\hline Fibrilação atrial & 1351 & $1.7 \%$ & $33.4 \%$ & 4.0 & 2.0 & 8.0 & $3 \%$ \\
\hline Infarto miocárdico sem supra de ST & 1211 & $1.5 \%$ & $34.9 \%$ & 5.2 & 3.0 & 10.0 & $4 \%$ \\
\hline Tromboembolismo pulmonar & 1155 & $1.4 \%$ & $36.4 \%$ & 5.0 & 3.0 & 8.0 & $3 \%$ \\
\hline Diagnosis Code with ICU LoS [0,1] & 5419 & $6.8 \%$ & $43.1 \%$ & 2.0 & 1.0 & 3.0 & $0 \%$ \\
\hline Diagnosis Code with ICU LoS ]1,2] & 15680 & $19.6 \%$ & $62.7 \%$ & 3.5 & 2.0 & 6.0 & $2 \%$ \\
\hline Diagnosis Code with ICU LoS ]2,3] & 14063 & $17.5 \%$ & $80.2 \%$ & 5.0 & 3.0 & 10.0 & $4 \%$ \\
\hline Diagnosis Code with ICU LoS ]3,4] & 8777 & $10.9 \%$ & $91.2 \%$ & 7.6 & 4.0 & 15.0 & $9 \%$ \\
\hline Diagnosis Code with ICU LoS ]4,5] & 2589 & $3.2 \%$ & $94.4 \%$ & 8.6 & 5.0 & 18.0 & $9 \%$ \\
\hline Diagnosis Code with ICU LoS ]5,7] & 1389 & $1.7 \%$ & $96.1 \%$ & 13.3 & 7.0 & 31.0 & $18 \%$ \\
\hline Diagnosis Code with ICU LoS >7 & 114 & $0.1 \%$ & $96.3 \%$ & 14.0 & 10.0 & 33.0 & $46 \%$ \\
\hline Others & 2996 & $3.7 \%$ & $100.0 \%$ & 6.0 & 3.0 & 14.0 & $7 \%$ \\
\hline
\end{tabular}




\section{Data Preparation}

- Feature engineering

Grouping "Admission - Main Diagnosis" (9 possible classes)

\begin{tabular}{crrrrrrr} 
Main Diagnosis Name & Total & \% & $\begin{array}{c}\text { Cumulati } \\
\text { ve \% }\end{array}$ & $\begin{array}{c}\text { Mean } \\
\text { ICU LoS }\end{array}$ & $\begin{array}{c}\text { Median } \\
\text { ICU LoS }\end{array}$ & $\begin{array}{c}\text { Q90 ICU } \\
\text { LoS }\end{array}$ & $\begin{array}{c}\text { ICU } \\
\text { Mortality }\end{array}$ \\
\hline$[0,1]$ & 5419 & $6.8 \%$ & $6.8 \%$ & 2.0 & 1.0 & 3.0 & $0.0 \%$ \\
\hline$] 1,2]$ & 26794 & $33.4 \%$ & $40.2 \%$ & 3.4 & 2.0 & 6.0 & $1.0 \%$ \\
\hline$[2,3]$ & 19679 & $24.5 \%$ & $64.7 \%$ & 5.3 & 3.0 & 10.0 & $4.0 \%$ \\
\hline$] 3,4]$ & 19105 & $23.8 \%$ & $88.5 \%$ & 7.7 & 4.0 & 16.0 & $9.0 \%$ \\
\hline 4,5$]$ & 4701 & $5.9 \%$ & $94.4 \%$ & 8.1 & 5.0 & 17.0 & $8.0 \%$ \\
\hline$[5,6]$ & 477 & $0.6 \%$ & $95.0 \%$ & 11.3 & 6.0 & 26.0 & $14.0 \%$ \\
\hline 6,7$]$ & 912 & $1.1 \%$ & $96.1 \%$ & 14.3 & 7.0 & 34.9 & $20.0 \%$ \\
\hline$>7$ & 114 & $0.1 \%$ & $96.3 \%$ & 14.0 & 10.0 & 33.0 & $46.0 \%$ \\
\hline Others & 2996 & $3.7 \%$ & $100.0 \%$ & 6.0 & 3.0 & 14.0 & $7.0 \%$ \\
\hline & & & & & & & \\
\hline
\end{tabular}




\section{Data Preparation}

- Feature engineering

Grouping "Admission - Main Diagnosis" (8 possible classes)

\begin{tabular}{crrrrrrrr} 
Main Diagnosis Name & Total & \% & $\begin{array}{c}\text { Cumulati } \\
\text { ve \% }\end{array}$ & $\begin{array}{c}\text { Mean } \\
\text { ICU LoS }\end{array}$ & $\begin{array}{c}\text { Median } \\
\text { ICU LoS }\end{array}$ & $\begin{array}{c}\text { Q90 ICU } \\
\text { LoS }\end{array}$ & $\begin{array}{c}\text { ICU } \\
\text { Mortality }\end{array}$ \\
\hline$[0,1]$ & 1064 & $1.3 \%$ & $1.3 \%$ & 1.7 & 1.0 & 2.8 & $0.0 \%$ \\
\hline$] 1,2]$ & 16623 & $20.9 \%$ & $22.2 \%$ & 2.5 & 1.7 & 4.7 & $1.0 \%$ \\
\hline$] 2,3]$ & 27129 & $34.1 \%$ & $56.3 \%$ & 3.8 & 2.6 & 7.7 & $2.0 \%$ \\
\hline$] 3,4]$ & 17201 & $21.6 \%$ & $77.9 \%$ & 5.4 & 3.6 & 12.7 & $6.0 \%$ \\
\hline$] 4,5]$ & 14860 & $18.7 \%$ & $96.6 \%$ & 6.7 & 4.6 & 17.1 & $10.0 \%$ \\
\hline$[5,6]$ & 1170 & $1.5 \%$ & $98.0 \%$ & 7.7 & 5.6 & 21.0 & $11.0 \%$ \\
{$[6,7]$} & 334 & $0.4 \%$ & $98.4 \%$ & 8.5 & 6.5 & 21.0 & $14.0 \%$ \\
\hline 7 & 1242 & $1.6 \%$ & $100.0 \%$ & 10.4 & 8.8 & 21.0 & $23.0 \%$ \\
\hline
\end{tabular}




\section{Data Preprocessing}

- Transformations of the training dataset to improve model performance.

- Dimension reduction

- Imputation

- Feature Selection

- Transformation to Resolve Skewness

- Normalization

- One-hot encoding 


\section{Data Preprocessing}

\section{- Dimension reduction}

- Zero and Near Zero Variance Predictors

- Vast majority of cases presenting a unique value and few cases presenting other values

- Identifying Correlated Predictors

- redundant predictors can add more complexity to the model than information

- for numeric variables, we used Pearson Correlation with a recommended threshold of 0.75

- for categorical, we employed Cramér's $\vee$ with a recommended threshold of 0.5 


\section{Results - Dimension reduction}

- Removing Zero and Near Zero Variance

- Demographic data:

- IsReadmission24h;IsReadmission48h

- Comorbidities:

- IsOtherSolidOrganTransplant

- IsAtrialFlutter

- IsCombinedPancreaskidneyTransplant

- IsHyperthyroidism

- IsAllogeneicBMT

- IsAutologousBMT

- IsPepticDisease

- ICU complications:

- IsNeutropenia

- IsVentricularSustainedCardiopulmonary

- IsCombinedLiverkidneyTransplant

- Laboratorial data:

- $\mathrm{PaO} 2 \mathrm{FiO} 2$ 


\section{Results - Dimension reduction}

- Removing Zero and Near Zero Variance

- Demographic data:

- IsReadmission24h;IsReadmission48h

- Comorbidities:

- IsOtherSolidOrganTransplant

- IsAtrialFlutter

- IsCombinedPancreaskidneyTransplant

- IsHyperthyroidism

- IsAllogeneicBMT

- IsAutologousBMT

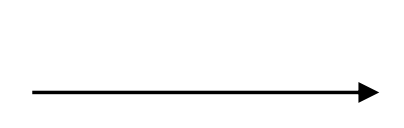

- IsPepticDisease

\begin{tabular}{|cccccccc} 
Feature & Class & Mean & SD & N & Beta & Cl & Pvalue \\
IsAutologousBMT & 0 & 5.35 & 10.83 & 80168 & ref. & - & - \\
\hline IsAutologousBMT & 1 & 6.38 & 8.42 & 29 & 1.03 & {$[-2.91,4.97]$} & 0.6094
\end{tabular}

- ICU complications:

- IsNeutropenia

- IsVentricularSustainedCardiopulmonary

- IsCombinedLiverkidneyTransplant

- Laboratorial data:

- $\mathrm{PaO} 2 \mathrm{FiO} 2$ 


\section{Results - Dimension reduction}

- Correlation for numeric features (collinearity analysis)

\begin{tabular}{llr} 
Feature 1 & Feature 2 & Correlation \\
\hline BUN & Urea & 1.00 \\
MFIScore & MFIpoints & 1.00 \\
Saps3DeathProbabilityStandardEquation & Saps3Points & 0.94 \\
LowestMeanArterialPressure1h & LowestDiastolicBloodPressure1h & 0.93 \\
LowestMeanArterialPressure1h & LowestSystolicBloodPressure1h & 0.89 \\
LowestDiastolicBloodPressure1h & LowestSystolicBloodPressure1h & 0.66 \\
SofaScore & Saps3DeathProbabilityStandardEquation & 0.64 \\
Saps3Points & Age & 0.63 \\
LowestGlasgowComaScale1h & SofaScore & -0.60
\end{tabular}

- Removed features (threshold=0.75):

- BUN; MFIpoints; Saps3DeathProbabilityStandardEquation; LowestDiastolicBloodPressure1h; LowestSystolicBloodPressure1h. 


\section{Results - Dimension reduction}

- Cramer-V for categorical features (collinearity analysis)

\begin{tabular}{llr} 
Feature 1 & Feature 2 & Correlation \\
\hline IsAsystole & IsCardiopulmonaryArrest & 0.72 \\
IsChemotherapy & IsImmunossupression & 0.70 \\
IsVasopressors & IsMechanicalVentilation & 0.61 \\
IsPulselessElectricalActivity & IsCardiopulmonaryArrest & 0.53 \\
has_complication & IsNonInvasiveVentilation & 0.50 \\
has_complication & IsRespiratoryFailure & 0.48 \\
has_complication & IsVasopressors & 0.47 \\
AdmissionSourceName & AdmissionReasonName & 0.47 \\
IsRadiationTherapy & IsChemotherapy & 0.44 \\
IsSteroidsUse & IsImmunossupression & 0.43 \\
has_complication & IsMechanicalVentilation & 0.43 \\
IsRadiationTherapy & IsImmunossupression & 0.42 \\
AdmissionMainDiagnosisName & AdmissionReasonName & 0.42
\end{tabular}

- Removed features (threshold=0.47):

IsCardiopulmonaryArrest; IsImmunossupression; AdmissionReasonName; has_complication 


\section{Data Preprocessing}

- Imputation

- Inadequate handling of missing data can lead to biased or inefficient estimates of parameters

- Missing at random (MAR) - the probability of data being missing does not depend on the unobserved data, conditional on the observed data

- Multiple imputation by chained equations (MICE)

- Missing not at random (MNAR) - the probability of data being missing does depend on the unobserved data, conditional on the observed data

- Laboratory variables: replacing missing values with the mean or median of the observed values 


\section{Data Preprocessing}

- Feature Selection

- Reduce the dimension of the problem by removing variables that do not present significant contribution to the model.

- Recursive Feature Elimination with random forest (RF-RFE),

- LASSO

- Selection by Filter (SBF) 


\section{Data Preprocessing}

- Transformation to Resolve Skewness

- Right skewed distribution can affect the performance of some classifiers

- Replacing that data with the log, square root, or inverse may help to remove the skew

- Box-cox transformations use maximum likelihood estimation to determine lambda in training data

$$
x^{*}= \begin{cases}\frac{x^{\lambda}-1}{\lambda} & \text { if } \lambda \neq 0 \\ \log (x) & \text { if } \lambda=0\end{cases}
$$

- Log transformation (lambda=0); square transformation (lambda $=2)$, square root (lambda $=$ $0.5)$, inverse (lambda $=-1)$, and others in-between. 


\section{Data Preprocessing}

- Normalization

- Set the numerical variables of the database on a common scale.

- Improve the numerical stability of some calculations.

- To scale the data between 0 and 1

- Min-Max Normalization or Normalization by Range:

$$
z_{i}=\frac{x_{i}-\min (x)}{\max (x)-\min (x)}
$$




\section{Data Preprocessing}

- One-hot encoding

- One-hot encoding is a method used to handle datasets with mixed data types (numerical, categorical and binary)

- Some machine learning prediction models does not accept this type of data.

- The method is used to encode a categorical feature with $\mathrm{k}$ possible values to $\mathrm{k}$ features.

- The feature representing the corresponding category has a value of 1 , and all other features have values of 0 . 


\section{Over-Fitting and Model Tuning}

- The hyper-parameters of the model will be selected in order to minimize the prediction error

- Measure of error:

- Root Mean Square Error (RMSE)

$$
R M S E=\sqrt{\sum_{i=1}^{n} \frac{\left(\hat{y}_{i}-y_{i}\right)^{2}}{n}}
$$




\section{Regression Models}

-We will test the following machine learning techniques:

- Support Vector Regression (SVR)

- CART

- Bagging

- Gradient Boosting Trees

- Random Forests

- k-nearest Neighborhood

- Linear Regression

- Glm with Negative Binomial distribution 


\section{Scenarios}

- Main Diagnosis

- 851 codes

- 19 grouped codes

- 9 grouped codes

- 8 grouped codes

- ICU length of stay:

- Raw

- Truncated

- Log-transformed

- Feature selection analysis

- All features

- Severity scores (MFI, SOFA, SAPS3) x Raw features (Comorbidities, Complications, and Laboratorial data)

- Missing data analysis

- Comparison between regression models 


\section{Results}

- Scenario 1: Comparison between "Main Diagnosis" groups

- Truncated LoS; All features; No feature selection; model = "boosting"

\begin{tabular}{|c|c|c|c|c|c|c|c|c|c|c|}
\hline \multirow[b]{2}{*}{ Type of grouping for "Main Diagnosis" } & \multicolumn{4}{|c|}{ Testing set } & \multicolumn{6}{|c|}{ Training set } \\
\hline & RMSE & MAE & $\mathbf{R}^{\mathbf{2}}$ & Cor & RMSE & (SD) & MAE & (SD) & $\mathbf{R}^{\mathbf{2}}$ & (SD) \\
\hline Original dataset with 851 diagnosis codes & 3.97 & 2.64 & $31 \%$ & 0.554 & 3.96 & 0.06 & 2.60 & 0.03 & $33 \%$ & 0.01 \\
\hline Dataset with 339 diagnosis codes (Diagnosis $<20=$ Others) & 3.97 & 2.64 & $31 \%$ & 0.556 & 3.96 & 0.03 & 2.60 & 0.01 & $32 \%$ & 0.01 \\
\hline Dataset with 19 grouped diagnosis (Diagnosis $<20=$ Others) & 3.97 & 2.64 & $31 \%$ & 0.554 & 3.96 & 0.03 & 2.60 & 0.01 & $32 \%$ & 0.00 \\
\hline Dataset with 9 grouped diagnosis (Diagnosis $<20=$ Others) & 4.00 & 2.66 & $30 \%$ & 0.546 & 3.97 & 0.04 & 2.60 & 0.01 & $32 \%$ & 0.01 \\
\hline Dataset with 8 grouped diagnosis & 3.99 & 2.65 & $30 \%$ & 0.550 & 3.96 & 0.03 & 2.59 & 0.01 & $32 \%$ & 0.01 \\
\hline
\end{tabular}




\section{Results}

- Scenario 1: Comparison between "Main Diagnosis" groups

- Truncated LoS; All features; No feature selection; model = "boosting"

Original dataset with 851 diagnosis $($ Cor $=0.554)$

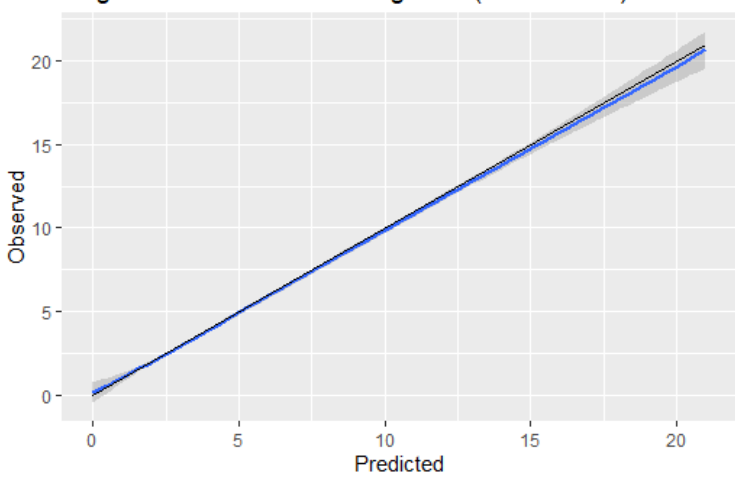

Dataset with 339 diagnosis $($ Cor $=0.556)$

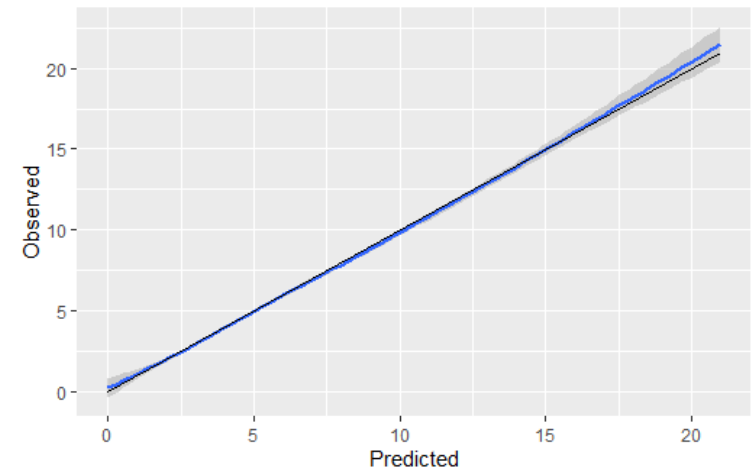

Dataset with 19 grouped diagnosis $(0.554)$

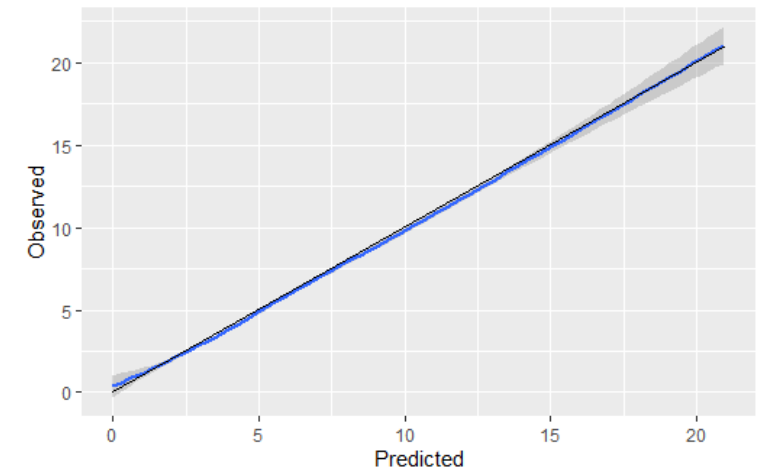

Dataset with 9 grouped diagnosis $($ Cor $=0.546)$

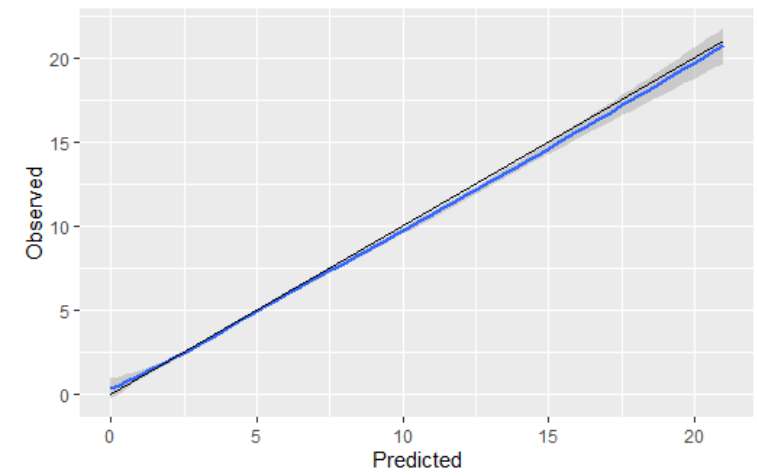

Dataset with 8 grouped diagnosis $(\mathrm{Cor}=0.550$ )

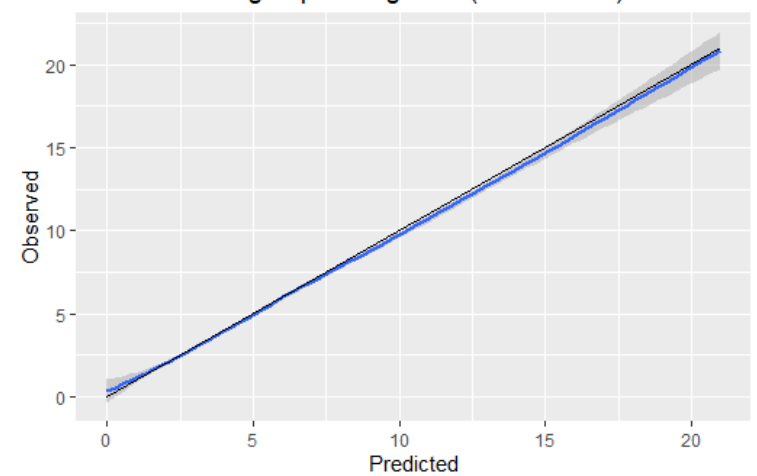




\section{Results}

- Scenario 2: Comparison between types of LoS

- All features; No feature selection; model = "boosting"; 8 diagnosis groups

- All models were compared truncating the ICU LoS bigger than 21 days (in the test set)

\begin{tabular}{lcccc} 
& \multicolumn{4}{c}{ Testing set } \\
Type of LoS & RMSE & MAE & $\mathbf{R}^{\mathbf{2}}$ & Cor \\
\hline Truncated LoS & $\mathbf{3 . 9 9}$ & $\mathbf{2 . 6 5}$ & $\mathbf{3 0 \%}$ & $\mathbf{0 . 5 5}$ \\
Original LoS & 4.05 & 2.69 & $28 \%$ & 0.54 \\
BoxCox LoS (Truncated) & 4.09 & 2.50 & $27 \%$ & 0.53 \\
BoxCox LoS (Original) & 4.15 & 2.61 & $13 \%$ & 0.50 \\
\hline
\end{tabular}




\section{Results}

- Scenario 2: Comparison between types of LoS

- All features; No feature selection; model = "boosting"; 8 diagnosis groups
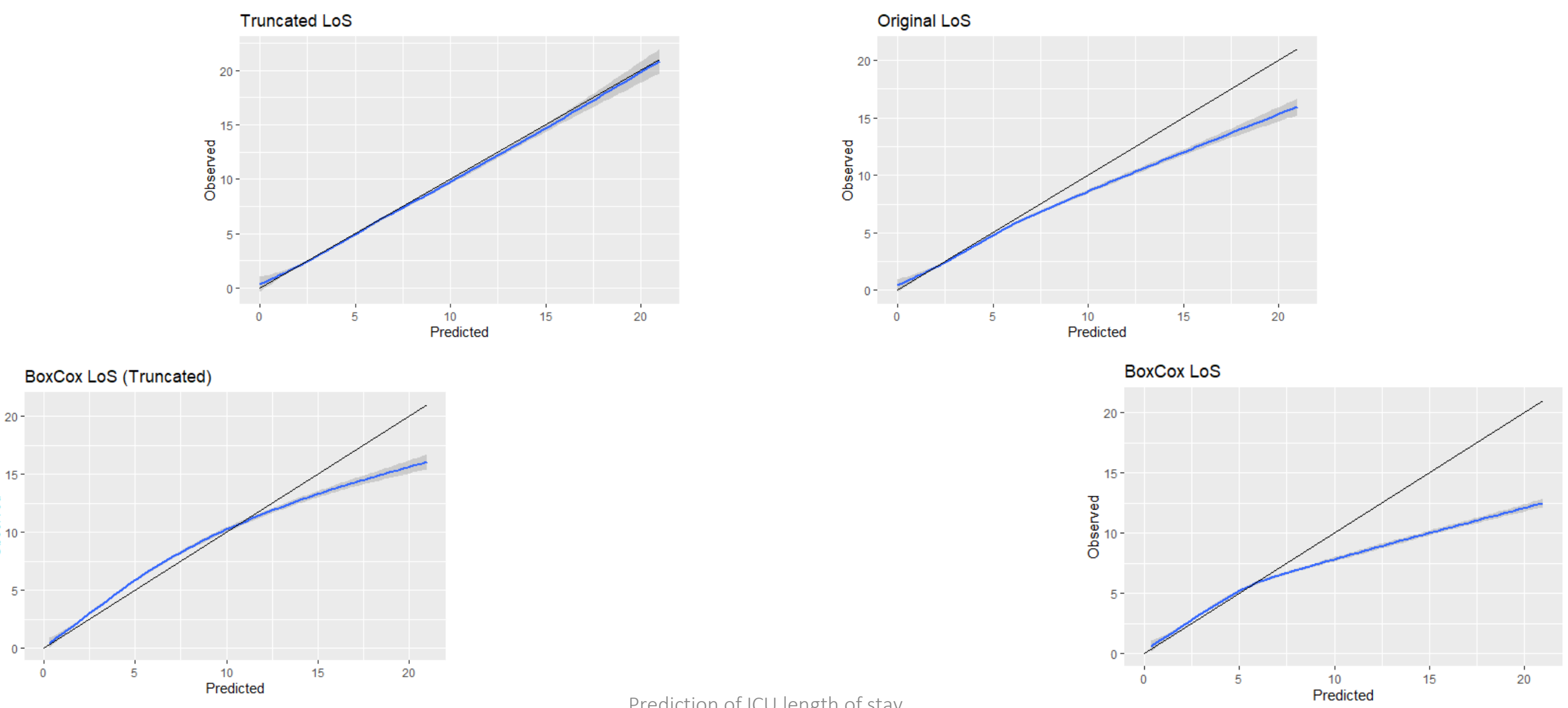


\section{Results}

\section{- Scenario 3: Severity scores, feature selection and missing data analyses}

- Truncated LoS; 8 diagnosis groups; model = "boosting"

- Severity scores = SAPS3, SOFA, MFI, Charlson Comorbidity Index

\begin{tabular}{|c|c|c|c|c|c|c|c|c|c|c|}
\hline \multirow{2}{*}{ Severity scores, feature selection and missing data analyses } & \multicolumn{4}{|c|}{ Testing set } & \multicolumn{6}{|c|}{ Training set } \\
\hline & RMSE & MAE & $\mathbf{R}^{2}$ & Cor & RMSE & (SD) & MAE & (SD) & $\mathbf{R}^{\mathbf{2}}$ & (SD) \\
\hline Dataset with all features (90 features) & 3.99 & 2.65 & $30 \%$ & 0.550 & 3.96 & 0.03 & 2.59 & 0.01 & $32 \%$ & 0.01 \\
\hline \multicolumn{11}{|l|}{ Severity scores analysis } \\
\hline Dataset considering only severity scores features ( 4 features) & 4.29 & 2.85 & $19 \%$ & 0.440 & 4.32 & 0.02 & 2.86 & 0.01 & $20 \%$ & 0.00 \\
\hline Dataset not considering severity scores features ( 86 features) & 4.01 & 2.67 & $29 \%$ & 0.544 & 3.97 & 0.04 & 2.61 & 0.03 & $32 \%$ & 0.01 \\
\hline \multicolumn{11}{|l|}{ Feature Selection analysis } \\
\hline Dataset after feature selection (14 features of Rfe Treebag) & 4.08 & 2.69 & $27 \%$ & 0.519 & 4.06 & 0.02 & 2.66 & 0.01 & $29 \%$ & 0.00 \\
\hline Dataset after feature selection ( 26 features of Rfe Treebag) & 4.03 & 2.68 & $29 \%$ & 0.536 & 3.99 & 0.03 & 2.61 & 0.01 & $31 \%$ & 0.01 \\
\hline Dataset after feature selection ( 27 features of Rfe Random Forest) & 4.03 & 2.68 & $29 \%$ & 0.537 & 3.99 & 0.03 & 2.61 & 0.01 & $32 \%$ & 0.00 \\
\hline Dataset after feature selection without severity scores ( 28 features of Rfe Treebag) & 4.03 & 2.68 & $29 \%$ & 0.537 & 3.99 & 0.03 & 2.62 & 0.01 & $31 \%$ & 0.00 \\
\hline $\begin{array}{l}\text { Dataset after feature selection without severity scores and glasgow } \\
\text { ( } 25 \text { features of Rfe Treebag) }\end{array}$ & 4.06 & 2.70 & $28 \%$ & 0.525 & 4.02 & 0.02 & 2.64 & 0.01 & $30 \%$ & 0.01 \\
\hline \multicolumn{11}{|l|}{ Missing data analysis } \\
\hline $\begin{array}{l}\text { Dataset after feature selection without features with more than } 15 \% \text { of missing } \\
\text { ( } 26 \text { features of Rfe Treebag) }\end{array}$ & 4.05 & 2.67 & $28 \%$ & 0.532 & 4.02 & 0.03 & 2.64 & 0.01 & $31 \%$ & 0.00 \\
\hline $\begin{array}{l}\text { Dataset after feature selection without features with more than } 3 \% \text { of missing } \\
\text { ( } 26 \text { features of Rfe Treebag) }\end{array}$ & 4.07 & 2.68 & $27 \%$ & 0.523 & 4.11 & 0.03 & 2.70 & 0.01 & $27 \%$ & 0.01 \\
\hline
\end{tabular}




\section{Results}

- Scenario 3: Severity scores and feature selection analysis

- Truncated LoS; 8 diagnosis groups; model = "boosting"

28 features of Rfe Treebag - No Scores

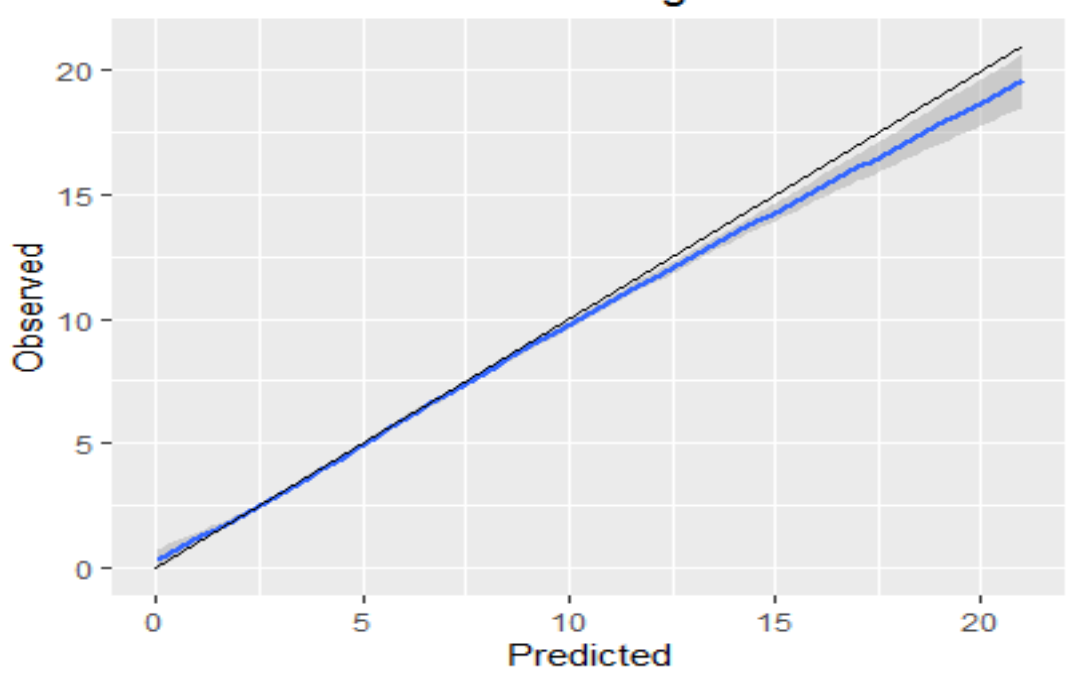




\section{Results}

- Scenario 4: Comparison of Regression Models

- Truncated LoS; 8 Diagnosis Groups; 28 selected features from RFE

\begin{tabular}{|c|c|c|c|c|c|c|c|c|c|c|}
\hline \multirow{2}{*}{ Models } & \multicolumn{4}{|c|}{ Testing set } & \multicolumn{6}{|c|}{ Training set } \\
\hline & RMSE & MAE & $\mathbf{R}^{2}$ & Cor & RMSE & (SD) & MAE & (SD) & $\mathrm{R}^{2}$ & (SD) \\
\hline Random Forest & 3.84 & 2.58 & 0.35 & 0.596 & 3.90 & 0.02 & 2.59 & 0.01 & 0.35 & 0.003 \\
\hline Boosting (GBM) & 4.03 & 2.68 & 0.29 & 0.537 & 3.99 & 0.03 & 2.62 & 0.01 & 0.31 & 0.005 \\
\hline Linear Regression & 4.16 & 2.77 & 0.24 & 0.491 & 4.19 & 0.02 & 2.77 & 0.01 & 0.25 & 0.003 \\
\hline kNN & 4.17 & 2.67 & 0.24 & 0.492 & 4.22 & 0.03 & 2.69 & 0.01 & 0.24 & 0.003 \\
\hline Glm Negative Binomial & 4.17 & 2.75 & 0.24 & 0.487 & 4.20 & 0.04 & 2.75 & 0.02 & 0.24 & 0.009 \\
\hline CART & 4.21 & 2.80 & 0.22 & 0.473 & 4.22 & 0.03 & 2.80 & 0.02 & 0.23 & 0.008 \\
\hline SVR Radial & 4.24 & 2.49 & 0.21 & 0.502 & 4.29 & 0.03 & 2.50 & 0.01 & 0.25 & 0.006 \\
\hline Bagging & 4.32 & 2.91 & 0.18 & 0.428 & 4.33 & 0.04 & 2.91 & 0.01 & 0.19 & 0.010 \\
\hline SVR Linear & 4.34 & 2.56 & 0.17 & 0.478 & 4.38 & 0.03 & 2.56 & 0.01 & 0.23 & 0.004 \\
\hline
\end{tabular}




\section{Results - Variable Importance}

- Random Forests

\begin{tabular}{|l|c|}
\hline Features & Importance \\
\hline LowestGlasgowComaScale1h & $100.00 \%$ \\
Urea & $70.97 \%$ \\
\hline AdmissionMainDiagnosisName & $68.22 \%$ \\
\hline Age & $53.56 \%$ \\
\hline IsMechanicalVentilation & $50.55 \%$ \\
\hline LengthHospitalStayPriorUnitAdmission & $41.10 \%$ \\
\hline HighestLeukocyteCount1h & $39.75 \%$ \\
\hline HighestCreatinine1h & $38.37 \%$ \\
BMI & $37.99 \%$ \\
\hline HighestHeartRate1h & $32.01 \%$ \\
\hline Bilirubin & $22.45 \%$ \\
\hline HighestTemperature1h & $19.34 \%$ \\
\hline HighestRespiratoryRate1h & $16.66 \%$ \\
\hline n_complication & $14.13 \%$ \\
\hline AdmissionSourceName & $14.06 \%$ \\
\hline AdmissionTypeName & $12.67 \%$ \\
IsVasopressors & $10.80 \%$ \\
\hline IsNonInvasiveVentilation & $9.10 \%$ \\
\hline IsDementia & $4.52 \%$ \\
\hline IsCrfNo & $3.59 \%$ \\
\hline FrailPatientMFI & $3.55 \%$ \\
\hline Gender & $2.93 \%$ \\
\hline ChfNyha & $2.11 \%$ \\
\hline IsAngina & $1.34 \%$ \\
\hline IsAcuteAtrialFibrilation & $0.87 \%$ \\
\hline IsAlcoholism & $0.85 \%$ \\
\hline IsAcuteKidneyInjury & $0.70 \%$ \\
\hline IsAids & $0.20 \%$ \\
\hline
\end{tabular}




\section{Conclusions}

Benefits of obtaining a good estimate for the length of stay:

- Assistance level:

- optimize the implementation of healthcare protocols (such as sedation and mobilization),

- discussion of the cases in each multi-professional round,

- better preparation of healthcare transit.

- Operational level:

- planning the ICU discharge,

- prioritizing patients to be evaluated daily,

- better communication between family members, teams, and managers.

- Strategic level:

- better sizing the number of beds,

- improving the benchmarking analysis between ICUs. 